\title{
AUTOMORPHISMS AND IDEALS OF THE WEYL ALGEBRA
}

\author{
YURI BEREST AND GEORGE WILSON
}

\begin{abstract}
Let $A_{1}$ be the (first) Weyl algebra, and let $G$ be its automorphism group. We study the natural action of $G$ on the space of isomorphism classes of right ideals of $A_{1}$ (equivalently, of finitely generated rank 1 torsion-free right $A_{1}$-modules). We show that this space breaks up into a countable number of orbits each of which is a finite dimensional algebraic variety. Our results are strikingly similar to those for the commutative algebra of polynomials in two variables; however, we do not know of any general principle that would allow us to predict this in advance. As a key step in the proof, we obtain a new description of the bispectral involution of |W1|. We also make some comments on the group $G$ from the viewpoint of Shafaravich's theory of infinite dimensional algebraic groups.
\end{abstract}

\section{IntroduCtion AND STATEMENT OF RESUlts}

The main aim of this paper is to give a simple description of the space $\mathcal{R}$ of isomorphism classes of (non-zero) right ideals in the complex Weyl algebra $A_{1}$, and of the action on $\mathcal{R}$ of the automorphism group of $A_{1}$. We begin by reviewing the corresponding (relatively trivial) results for the commutative algebra $A_{0}=\mathbb{C}[x, y]$.

We call two non-zero ideals in $A_{0}$ isomorphic if they are isomorphic as $A_{0^{-}}$ modules; equivalently, ideals $I$ and $J$ are isomorphic if we have $p I=q J$ for some $p, q \in A_{0}$. Using the fact that reflexive ideals in $A_{0}$ are principal, it is easy to show that each isomorphism class contains a unique ideal of finite codimension. The ideals of a fixed codimension $n$ form a well-studied space, the Hilbert scheme $\operatorname{Hilb}_{n}\left(\mathbb{A}^{2}\right)$ of $n$-points in the affine plane. Alternatively, we can think of this space as the space of isomorphism classes of $n$-dimensional cyclic representations of $A_{0}$ : to an ideal $I$ we assign the natural representation of $A_{0}$ on the quotient space $V=A_{0} / I$. A representation of $A_{0}$ on $V$ is nothing but a pair of commuting endomorphisms of $V$ (corresponding to the action of the generators $x$ and $y$ of $\left.A_{0}\right)$; choosing a basis for $V$, we find that $I$ is represented by a pair of commuting matrices $(X, Y)$, uniquely determined up to simultaneous conjugation. Now let $\sigma$ be an automorphism of $A_{0}$ : it induces an isomorphism (of rings) $\hat{\sigma}: A_{0} / I \rightarrow A_{0} / \sigma(I)$. If we use $\hat{\sigma}$ to identify these two spaces, and choose a basis, we find that the pair of matrices corresponding to $\sigma(I)$ is just $\left(\sigma^{-1}(X), \sigma^{-1}(Y)\right)$ (the notation means that we take the polynomials $\sigma^{-1}(x), \sigma^{-1}(y) \in A_{0}$ and substitute $(X, Y)$ for $\left.(x, y)\right)$. To summarize:

1. The space of isomorphism classes of ideals breaks up into the disjoint union of the Hilbert schemes $\operatorname{Hilb}_{n}\left(\mathbb{A}^{2}\right)$.

The second author was supported in part by NSF Grant DMS-94-00097; he wishes to thank MSRI and the University of California at Berkeley for their generous hospitality during the academic year 1998/9. 
2. A point of $\operatorname{Hilb}_{n}\left(\mathbb{A}^{2}\right)$ can be identified with a conjugacy class of pairs $(X, Y)$ of commuting $n \times n$ matrices (possessing a cyclic vector).

3. The formula for the action of an automorphism $\sigma$ on the pair of matrices $(X, Y)$ is the same as the formula for the action of $\sigma^{-1}$ on the generators $(x, y)$ of $A_{0}$.

Note that, although the proofs of these statements are almost trivial, they all depend crucially on the first step, reducing the problem to the case of ideals of finite codimension.

Now let us turn to the Weyl algebra $A_{1}$. We recall that $A_{1}$ is the associative algebra (over $\mathbb{C}$ ) generated by two elements $x$ and $y$ subject to the single relation $[x, y]-1=0$. In the main body of the paper we shall use the well known realization of $A_{1}$ as the algebra of ordinary differential operators with polynomial coefficients; in this Introduction we think of $A_{1}$ more abstractly, as a noncommutative version of $A_{0}$. A fundamental difference between $A_{0}$ and $A_{1}$ is that $A_{1}$ has no finite dimensional representations (of positive dimension). Indeed, if $X$ and $Y$ are two $n \times n$ matrices with $[X, Y]-\mathrm{I}=0$, then taking the trace, we obtain $n=0$. Similarly, $A_{1}$ has no (left or right) ideals $I$ of finite codimension (for if it did, the quotient $A_{1} / I$ would be a finite dimensional representation). Thus, a priori, there seems no reason to expect that there should be results for $A_{1}$ similar to the ones indicated above for $A_{0}$ : nevertheless, we shall see that this is the case. To obtain such results, we have to relax somewhat the notion of a representation, as follows. For each $n \geq 0$, let $\mathcal{C}_{n}$ be the space of equivalence classes (modulo simultaneous conjugation) of pairs $(X, Y)$ of $n \times n$ matrices satisfying the condition

$$
[X, Y]-\mathrm{I} \text { has rank at most } 1
$$

(the mysterious "at most" takes care of the case $n=0$ ). We might think of $\mathcal{C}_{n}$ as the space of "approximate $n$-dimensional representations" of $A_{1}$. Let

$$
\mathcal{C}=\bigsqcup_{n \geq 0} \mathcal{C}_{n}
$$

be the disjoint union of the spaces $\mathcal{C}_{n}$. In W2], one of the authors constructed a bijective map

$$
\beta: \mathcal{C} \rightarrow \mathrm{Gr}^{\text {ad }}
$$

from $\mathcal{C}$ to the adelic Grassmannian that parametrizes rational solutions of the KP hierarchy. This Grassmannian arises (thinly disguised) also in the work of Cannings and Holland on ideals in $A_{1}$. As for $A_{0}$, two right ideals $I$ and $J$ are called isomorphic if they are isomorphic as right $A_{1}$-modules; equivalently, if we have $p I=q J$ for some $p, q \in A_{1}$. Let $\mathcal{R}$ denote the set of isomorphism classes of nonzero right ideals. In [CH], Cannings and Holland constructed (in effect) a bijective map

$$
\alpha: \mathrm{Gr}^{\mathrm{ad}} \rightarrow \mathcal{R} .
$$

Set $\omega=\alpha \beta$. Combining the results of [CH] and [W2], we obtain at once

Theorem 1.1. The map $\omega: \mathcal{C} \rightarrow \mathcal{R}$ is bijective.

Now let $G$ be the group of automorphisms of $A_{1}$. The natural action of $G$ on $A_{1}$ induces an action on $\mathcal{R}$ (for if $I$ is a right ideal of $A_{1}$ and $\sigma \in G$, then the isomorphism class of the right ideal $\sigma(I)$ clearly depends only on that of $I$ ). We 
transfer this action to $\operatorname{Gr}^{\text {ad }}$ via the bijection $\alpha$, and then to $\mathcal{C}$ via the bijection $\beta$. To describe the resulting action of $G$ on $\mathcal{C}$, recall (see [D], [M2]) that $G$ is generated by the special automorphisms of the form

$$
\Phi_{p}(D)=e^{p(y)} D e^{-p(y)}, \quad \Psi_{q}(D)=e^{q(x)} D e^{-q(x)},
$$

where $p$ and $q$ run over all polynomials (say without constant term). The action of these automorphisms on the generators $x$ and $y$ of $A_{1}$ is given by

$$
\begin{aligned}
& \Phi_{p}(x)=x-p^{\prime}(y), \quad \Phi_{p}(y)=y \\
& \Psi_{q}(x)=x, \quad \Psi_{q}(y)=y+q^{\prime}(x) .
\end{aligned}
$$

We shall prove

Theorem 1.2. The action of $G$ on $\mathcal{C}$ preserves each of the subspaces $\mathcal{C}_{n}$. The action of the generators of $G$ on $\mathcal{C}_{n}$ is given by the formulae

$$
\begin{gathered}
\Phi_{p}(X, Y)=\left(X+p^{\prime}(Y), Y\right) \\
\Psi_{q}(X, Y)=\left(X, Y-q^{\prime}(X)\right) .
\end{gathered}
$$

Theorem 1.3. The action of $G$ on each of the spaces $\mathcal{C}_{n}$ is transitive; that is, the $\mathcal{C}_{n}$ are the orbits of the action of $G$ on $\mathcal{C}$.

Theorem 1.3 is perhaps particularly unexpected, because it is a simpler result than we have in the commutative case. Indeed, the automorphism group of $\mathbb{C}[x, y]$, or, equivalently, of the affine plane $\mathbb{A}^{2}$, does not act transitively on $\operatorname{Hilb}_{n}\left(\mathbb{A}^{2}\right)$ for $n>1$, because any automorphism preserves the multiplicities of a collection of $n$ points of $\mathbb{A}^{2}$. See the last section of the paper for a further comment on this matter.

Of course, it follows at once from Theorem 1.3 that the spaces $\beta\left(\mathcal{C}_{n}\right)$ are the orbits of the action of $G$ on $\mathrm{Gr}^{\text {ad }}$; and that the spaces $\omega\left(\mathcal{C}_{n}\right)$ are the orbits of the action of $G$ on $\mathcal{R}$. Each of these facts has considerable independent interest. In W2 the spaces $\beta\left(\mathcal{C}_{n}\right)$ were characterized as the union of all the $n$-dimensional open cells in $\mathrm{Gr}^{\text {ad }}$; but our present description of them as the orbits of a group action seems more straightforward. The problem of describing the orbits of $G$ in $\mathcal{R}$ goes back at least to Stafford's paper [St2]: it is known to be equivalent to the problem of classifying rings of differential operators on affine curves; or again, to classifying the algebras Morita equivalent to $A_{1}$ (see, for example, [BW], [CH], [K], [St2], W3]). The key fact that the $G$-orbits in $\mathcal{R}$ are classified by a single nonnegative integer $n$ was first discovered in the 1994 thesis [K] of Kouakou: however, Kouakou's work was not published at that time, and remained unknown to us until after we rediscovered his main result in [BW].

Here are a few words about our proof of Theorem 1.2. The difficulty is that the maps $\alpha$ and $\beta$ do not respect the symmetry between $x$ and $y$ (or $X$ and $Y$ ), so that although the formula (1.6) is easy to check, the similar formula (1.7) is not: indeed, we shall see that the automorphisms $\Phi_{p}$ act on $\mathrm{Gr}^{\text {ad }}$ as the group of KP flows, whereas we do not know any such simple description of the action of the $\Phi_{q}$. To pinpoint the problem, we introduce the formal Fourier transform $\varphi$ : it is the automorphism of $A_{1}$ whose action on the generators is given by

$$
\varphi(x)=-y, \varphi(y)=x .
$$


We have $\Psi_{p}=\varphi \Phi_{p} \varphi^{-1}$, hence the $\Phi_{p}$ and $\varphi$ generate $G$; thus our problem is reduced to tracking how $\varphi$ acts on $\mathrm{Gr}^{\text {ad }}$, and thence on $\mathcal{C}$. Although the action of $\varphi$ on $\mathrm{Gr}^{\text {ad }}$ does not seem to have any simple description, we can write $\varphi$ as the composition $\varphi=b c$ of two involutory anti-automorphisms of $A_{1}$ : the action of $b$ and $c$ on the generators of $A_{1}$ is defined by

$$
b(x)=y, b(y)=x ; \quad c(x)=-x, c(y)=y .
$$

(If we think of $A_{1}$ as an algebra of differential operators, then $c$ is the formal adjoint). Using the fact that the ideals in $A_{1}$ are reflexive $A_{1}$-modules, we can extend the action of $G$ on $\mathcal{R}$ to an action of the larger group $\widehat{G}$ of automorphisms and anti-automorphisms of $A_{1}$; we then find that the actions of $b$ and $c$ on $\mathrm{Gr}^{\text {ad }}$ correspond to known involutions. In particular, we shall see

Theorem 1.4. The anti-automorphism b acts on $\mathrm{Gr}^{\text {ad }}$ as the bispectral involution introduced in $\mathrm{W} 1$.

Theorem 1.4 is (to us) the most difficult result in this paper: in the following Sections 2-8 we prepare the machinery for its proof, and also summarize the most essential definitions and facts that we need from the literature. The new material in these sections concerns some spaces of differential operators which we denote by $\mathcal{D}(U, V)$. Here $U$ and $V$ are certain linear subspaces of the space $\mathbb{C}(z)$ of rational functions in one variable, and $\mathcal{D}(U, V) \subset \mathbb{C}(z)[\partial / \partial z]$ is the set of all differential operators with rational coefficients that map $U$ into $V$ (mostly $U$ and $V$ will represent points of the Grassmannian $\left.\mathrm{Gr}^{\text {ad }}\right)$. These spaces $\mathcal{D}(U, V)$ play a basic role in the paper of Cannings and Holland, but (to our knowledge) they have not so far appeared in the theory of integrable systems. In that theory, on the other hand, an essential part of the machinery involves the Baker functions $\psi_{U}$ of points $U \in \mathrm{Gr}^{\text {ad }}$ : these functions do not appear in the work of Cannings and Holland. We bridge this gap in Section 8, which gives a description of $\mathcal{D}(U, V)$ in terms of the Baker functions of $U$ and $V$. Our main theorems all follow easily from this (and other facts available in the literature): the proofs are given in Sections 9 and 10. Finally, in the last section of the paper we offer a preliminary attempt to interpret our results from the perspective of algebraic groups.

In connection with Theorem 1.1, there are at least two natural questions which remain untouched in the present paper: one is to find a more direct definition of the map $\omega$ (without passing through the Grassmannian, where some of the symmetry is difficult to see), another is to describe explicitly its inverse. We shall address both these problems in our subsequent paper [BW1], where the correspondence $\omega$ will be constructed by cohomological methods developed recently in the framework of noncommutative algebraic geometry. The idea of such an approach goes back to the work of L. Le Bruyn [L]. In [BW1] we hope also to clarify the relationship between our stratification of $\mathcal{R}$ and Le Bruyn's "moduli spaces".

With the exception of Theorem 1.2, the main results of this article were announced in W3.

\section{The group $\Gamma$ And the spaces $\mathcal{D}(U, V)$}

In this section we confront the following technical problem: a point $W$ of $\mathrm{Gr}^{\text {ad }}$ is a (certain kind of) linear space of rational functions, but we need to consider the

action on $\mathrm{Gr}^{\text {ad }}$ of the group $\Gamma$ of KP flows. Loosely speaking, this action is given 
by multiplication by exponential functions. The most natural way to make sense of that is to work temporarily with the completion of $W$ in some larger function space. Because we need to consider also the action of $\Gamma$ on the spaces of differential operators $\mathcal{D}(U, V)$ it is inconvenient to use $\mathrm{L}^{2}$ completions (as in W1, [W2]); instead, we shall work with holomorphic functions, adapting to our circumstances a suggestion of ACKP].

To begin with, we work in slightly greater generality than will be needed for the main part of the paper. Let $\mathcal{S}$ be the set of all linear subspaces $V \subset \mathbb{C}[z]$ such that we have

$$
\rho(z) \mathbb{C}[z] \subset V \text { for some polynomial } \rho ;
$$

and let $\Gamma$ be the group of all functions of the form $\gamma(z)=e^{p(z)}$, where $p$ is a polynomial. To define the action of $\Gamma$ on $\mathcal{S}$, we introduce the space $\mathcal{H}$ of entire functions on $\mathbb{C}$, with its usual topology (uniform convergence on compact subsets), and denote by $\overline{\mathcal{S}}$ the set of all closed subspaces $\mathcal{V} \subset \mathcal{H}$ such that we have

$$
\rho(z) \mathcal{H} \subset \mathcal{V} \text { for some polynomial } \rho \text {. }
$$

If $V \in \mathcal{S}$, let $\bar{V}$ be the closure of $V$ in $\mathcal{H}$; then clearly, $\bar{V} \in \overline{\mathcal{S}}$. If $\mathcal{V} \in \overline{\mathcal{S}}$, we set $\mathcal{V}^{\text {alg }}=\mathcal{V} \cap \mathbb{C}[z]$; clearly, $\mathcal{V}^{\text {alg }} \in \mathcal{S}$. If $\rho$ is a polynomial of degree $n$, then we have the decomposition

$$
\mathcal{H}=\rho \mathcal{H} \oplus\{\text { polynomials of degree less than } n\}
$$

of $\mathcal{H}$ into a direct sum of two closed subspaces. Comparing this with the similar decomposition of $\mathbb{C}[z]$, we easily find

Lemma 2.1. The maps $V \mapsto \bar{V}$ and $\mathcal{V} \mapsto \mathcal{V}^{\text {alg }}$ define inverse bijections between $\mathcal{S}$ and $\overline{\mathcal{S}}$.

We use this Lemma to transfer the obvious action of $\Gamma$ on $\overline{\mathcal{S}}$ to $\mathcal{S}$; that is, if $V \in \mathcal{S}$ and $\gamma \in \Gamma$, we define

$$
\gamma V=(\gamma \bar{V})^{\mathrm{alg}}
$$

As in the Introduction, if $U, V \in \mathcal{S}$, we write

$$
\mathcal{D}(U, V)=\{D \in \mathbb{C}(z)[\partial]: D . U \subset V\}
$$

for the set of all differential operators with rational coefficients that map $U$ into $V$. Here $\partial \equiv \partial / \partial z$; we write $D . f$ for the action of $D$ on the function $f$ (to avoid confusion with the composition $D f$ of $D$ with the multiplication operator $f$ ). If $\rho$ is a polynomial, then we clearly have

$$
\mathcal{D}(\rho U, \rho V)=\rho \mathcal{D}(U, V) \rho^{-1} .
$$

We need to know that this is still true if we replace $\rho$ by an element of $\Gamma$.

Lemma 2.2. Let $U, V \in \mathcal{S}, D \in \mathbb{C}(z)[\partial]$. Then

$$
D . U \subset V \Longleftrightarrow D \cdot \bar{U} \subset \bar{V} .
$$

Proof. The implication " $\Leftarrow$ " is trivial. Conversely, suppose $D . U \subset V$, and choose a polynomial $\pi$ such that $\pi D$ has polynomial coefficients. Then $\pi D \cdot U \subset \pi V$; since $\pi D$ is a bounded operator on $\mathcal{H}$, it follows that $\pi D \cdot \bar{U} \subset \overline{\pi V}=\pi \bar{V}$. Hence $D \cdot \bar{U} \subset \bar{V}$, as required. 
Corollary 2.3. Let $U, V \in \mathcal{S}, \gamma \in \Gamma$. Then

$$
\mathcal{D}(\gamma U, \gamma V)=\gamma \mathcal{D}(U, V) \gamma^{-1} .
$$

Proof. The corresponding result for the closures $\bar{U}, \bar{V}$ is trivial, so the Corollary follows at once from Lemma 2.2 and the fact that the operators in $\gamma \mathcal{D}(U, V) \gamma^{-1}$ have rational coefficients.

\section{The Cannings-Holland correspondence}

For the rest of this paper we confine our attention to the spaces $V \in \mathcal{S}$ that are (in the terminology of [CH]) primary decomposable. The definition is as follows. First, if $\lambda \in \mathbb{C}$, a space $V \in \mathcal{S}$ is called $\lambda$-primary if the polynomial $\rho$ in (2.1) can be chosen to be a power of $z-\lambda$, that is, if $V$ contains the ideal $(z-\lambda)^{r} \mathbb{C}[z]$ for some $r \geq 0$. Then $V$ is called primary decomposable if it is a finite intersection of $\lambda$-primary subspaces (for various $\lambda$ ). The action of $\Gamma$ on $\mathcal{S}$ preserves the class of primary decomposable subspaces (for it clearly preserves the class of $\lambda$-primary subspaces for any fixed $\lambda$ ). Primary decomposable subspaces play a fundamental role in both of the papers [CH] and [W1]: indeed, this observation was the starting point for the present work.

To formulate the results of Cannings and Holland, we think of the Weyl algebra $A_{1}$ as the ring $\mathbb{C}[z, \partial / \partial z]$ of ordinary differential operators with polynomial coefficients. If $V \in \mathcal{S}$, we assign to it the right ideal

$$
\alpha(V)=\mathcal{D}(\mathbb{C}[z], V)
$$

of $A_{1}$. If $I$ is a right ideal in $A_{1}$, we assign to it the linear subspace $e(I)$ spanned by all polynomials of the form $D$. $f$ with $D \in I, f \in \mathbb{C}[z]$. Equivalently, we have simply

$$
e(I)=\{D .1: D \in I\} \subset \mathbb{C}[z] .
$$

The following theorem is proved in $[\mathrm{CH}]$.

Theorem 3.1. The maps $\alpha$ and e define inverse bijections between the set of primary decomposable subspaces of $\mathbb{C}[z]$ and the set of right ideals of $A_{1}$ that have non-zero intersection with $\mathbb{C}[z]$.

Now, it is easy to show that every non-zero ideal in $A_{1}$ is isomorphic (as right $A_{1}$-module) to one that intersects $\mathbb{C}[z]$ (see [St2], Lemma 4.2). Further, two ideals $I$ and $J$ that intersect $\mathbb{C}[z]$ are isomorphic if and only if $\pi(z) I=\rho(z) J$ for some polynomials $\pi$ and $\rho$. The maps $\alpha$ and $e$ commute with left multiplication by polynomials, so two primary decomposable subspaces $U$ and $V$ correspond to isomorphic ideals if and only if $\pi(z) U=\rho(z) V$ for some $\pi$ and $\rho$. Thus Theorem 3.1 implies the following

Corollary 3.2. The maps $\alpha$ and e determine inverse bijections between the set $\mathcal{R}$ of isomorphism classes of non-zero right ideals of $A_{1}$ and the set of classes of primary decomposable subspaces of $\mathbb{C}[z]$ modulo the equivalence relation

$$
U \equiv V \Longleftrightarrow \pi(z) U=\rho(z) V \text { for some polynomials } \pi \text { and } \rho \text {. }
$$




\section{Duality and the ACtion of $\widehat{G}$ ON $\mathcal{R}$}

Let $Q$ denote the quotient (skew) field of $A_{1}$. We recall that a right $A_{1}$-submodule $I$ of $Q$ is called a fractional right ideal if there is some $a \in A_{1}$ such that $a I \subset A_{1}$ (and hence $a I$ is a right ideal in the usual sense). Fractional left ideals are defined similarly. If $I$ is a fractional right ideal, its dual $I^{\prime}$ is defined by

$$
I^{\prime}=\left\{q \in Q: q I \subset A_{1}\right\} .
$$

Clearly, $I^{\prime}$ is a (fractional) left ideal; the terminology "dual" is justified by the easy

Lemma 4.1. As left $A_{1}$-module, $I^{\prime}$ is isomorphic to $\operatorname{Hom}_{A_{1}}\left(I, A_{1}\right)$.

Similarly, if $J$ is a (fractional) left ideal, the dual right ideal is defined by

$$
J^{\prime}=\left\{q \in Q: J q \subset A_{1}\right\} .
$$

A basic property of $A_{1}$ (see [MR]) is that it is a hereditary Noetherian ring, which means that its ideals are projective, and hence reflexive, as $A_{1}$-modules. That gives us the important

Lemma 4.2. For any right ideal $I$ and left ideal $J$, we have

$$
\left(I^{\prime}\right)^{\prime}=I \quad, \quad\left(J^{\prime}\right)^{\prime}=J .
$$

Using Lemma 4.2, we can extend the obvious action of $G=\operatorname{Aut}\left(A_{1}\right)$ on $\mathcal{R}$ to an action of the larger group $\widehat{G}$ of all automorphisms and anti-automorphisms of $A_{1}$. Recall from the Introduction that if $\sigma \in G$ is an automorphism of $A_{1}$, then we make $\sigma$ act on (the class of) the right ideal $I$ by

$$
\sigma \star I=\sigma(I) \equiv\{\sigma(i): i \in I\} .
$$

If now $\sigma$ is an anti-automorphism of $A_{1}$ and $I$ is a right ideal, then the set $\sigma(I)$ is a left ideal, so its dual is again a right ideal; we define the action of $\sigma$ on (the class of) $I$ by

$$
\sigma \star I=\sigma(I)^{\prime} \text { if } \sigma \text { is an anti-automorphism . }
$$

Lemma 4.3. The formulae (4.1) and (4.2) define an action of $\widehat{G}$ on $\mathcal{R}$.

Proof. Everything is trivial, except possibly to check that if $\sigma$ and $\tau$ are antiautomorphisms, and $\theta=\sigma \tau$, then $\sigma \star(\tau \star I)=\theta \star I$. For this, we need to prove that

$$
\sigma\left[\tau(I)^{\prime}\right]^{\prime}=\sigma[\tau(I)] .
$$

But because of Lemma 4.2, it is equivalent to prove that

$$
\sigma\left[\tau(I)^{\prime}\right]=\sigma[\tau(I)]^{\prime},
$$

which is easy.

\section{The adelic Grassmannian}

There does not seem to be any natural way of choosing representatives for the equivalence classes of primary decomposable subspaces in Corollary 3.2. However, these classes are in 1-1 correspondence with the points of the adelic Grassmannian $\mathrm{Gr}^{\text {ad }}$ introduced (for a different purpose) in W1. Let $V=\bigcap V_{\lambda}$ be the intersection of the $\lambda$-primary subspaces $V_{\lambda}$; let $k_{\lambda}$ be the codimension (in $\mathbb{C}[z]$ ) of $V_{\lambda}$, and set 


$$
m_{V}(z)=\prod_{\lambda}(z-\lambda)^{k_{\lambda}}
$$

Let $W=m_{V}^{-1} V$, so that $W$ is a linear subspace of $\mathbb{C}(z)$. By definition, $\mathrm{Gr}^{\text {ad }}$ consists of all subspaces $W \subset \mathbb{C}(z)$ that arise in this way. If we replace $V$ by $\rho V$ for some polynomial $\rho$, then $m_{V}$ gets replaced by $\rho m_{V}$; hence indeed the map $V \mapsto W$ induces a bijection from equivalence classes of primary decomposable subspaces to $\mathrm{Gr}^{\text {ad }}$. Note that the action of the group $\Gamma$ is compatible with the equivalence relation (3.1), and so induces an action on $\mathrm{Gr}^{\text {ad }}$. We write this action again as $W \mapsto \gamma W$, and think of it as scalar multiplication. If $U$ and $V$ are two points of $\mathrm{Gr}^{\text {ad }}$, we define the set of differential operators $\mathcal{D}(U, V)$ as before (see (2.2)).

Let us reformulate the Cannings-Holland results in terms of $\mathrm{Gr}^{\text {ad }}$. If $W \in \mathrm{Gr}^{\text {ad }}$, we set

$$
R_{W}=\mathcal{D}(\mathbb{C}[z], W) \equiv\{D \in \mathbb{C}(z)[\partial]: D \cdot \mathbb{C}[z] \subset W\} .
$$

Clearly, $R_{W}$ is is a fractional right ideal of $A_{1}$ (we regard $\mathbb{C}(z)[\partial]$ as a subalgebra of the quotient field of $\left.A_{1} \equiv \mathbb{C}[z][\partial]\right)$. Let $\alpha(W) \in \mathcal{R}$ be the isomorphism class of $R_{W}$; then Corollary 3.2 implies immediately

Theorem 5.1. The map $\alpha: \mathrm{Gr}^{\text {ad }} \rightarrow \mathcal{R}$ is bijective; that is, every non-zero right ideal of $A_{1}$ is isomorphic to a unique ideal of the form $R_{W}$. We can recover $W$ from $R_{W}$ by the formula

$$
W=e\left(R_{W}\right) \equiv\left\{D .1: D \in R_{W}\right\} .
$$

It is clear that the result of Corollary 2.3 is still valid for $U, V \in \mathrm{Gr}^{\text {ad }}$. Applying it with $U=\mathbb{C}[z], V=W$, we find that

$$
R_{\gamma W}=\gamma R_{W} \gamma^{-1} \text { for any } W \in \mathrm{Gr}^{\mathrm{ad}}, \gamma \in \Gamma .
$$

In other words, setting $\gamma(z)=e^{p(z)}$, we have

Proposition 5.2. Under the bijection $\alpha$, the action of the automorphism $\Phi_{p}$ on $\mathcal{R}$ corresponds to the map $W \mapsto e^{p(z)} W$ on $\mathrm{Gr}^{\mathrm{ad}}$.

We can also assign to each point $W$ of $\mathrm{Gr}^{\text {ad }}$ the fractional left ideal

$$
L_{W}=\mathcal{D}(W, \mathbb{C}[z]) \equiv\{D \in \mathbb{C}(z)[\partial]: D . W \subset \mathbb{C}[z]\} .
$$

This ideal is useful for studying the action of anti-automorphisms of $A_{1}$ on $\mathrm{Gr}^{\text {ad }}$, because we have

Lemma 5.3. The ideals $R_{W}$ and $L_{W}$ are dual to each other; that is, in the notation of the previous section, we have $L_{W}=\left(R_{W}\right)^{\prime}$.

Proof. It is obvious that $L_{W} \subset\left(R_{W}\right)^{\prime}$. The converse is a consequence of (5.2). Let $w \in W$ : then $w=D .1$ for some $D \in R_{W}$, so if $q \in\left(R_{W}\right)^{\prime}$, then $q \cdot w=(q D) .1$ is a polynomial, since $q D \in A_{1}$. Hence $q \in L_{W}$.

\section{The BAKer FunCtion}

Associated to each point $W$ of $\mathrm{Gr}^{\text {ad }}$ is the Baker function $\psi_{W}$. This function plays an indispensable role, because both the bispectral involution $b$ on $\mathrm{Gr}^{\text {ad }}$ and the map $\beta$ in 1.2 ) are defined in term of $\psi_{W}$. In this section we review briefly the definition and the main properties of $\psi_{W}$; the reader may consult [SW, W1, or W2] for more details. 
Let $R_{-}$be the space of rational functions that vanish at infinity, so that we have $\mathbb{C}(z)=\mathbb{C}[z] \oplus R_{-}$. If $W \in \mathrm{Gr}^{\text {ad }}$, then the projection $\pi_{W}: W \rightarrow \mathbb{C}[z]$ defined by this splitting is an operator of index zero (that is, its kernel and cokernel have the same (finite) dimension); we say that $W$ belongs to the big cell of $\mathrm{Gr}^{\text {ad }}$ if $\pi_{W}$ is an isomorphism. It is intuitively clear that "most" spaces $W$ belong to the big cell, in particular, that for any $W$, the space $\gamma W$ should belong to the big cell for almost all $\gamma \in \Gamma$. We need the following more precise result, which is a consequence of Lemma 8.6 in SW.

Proposition 6.1. For any $W \in \mathrm{Gr}^{\mathrm{ad}}$, the space $e^{x z} W$ belongs to the big cell for all but a discret 1 set of values of $x \in \mathbb{C}$.

It follows that for any $W$ there is a unique function $\tilde{\psi}_{W}(\gamma, z)$ (defined for almost all $\gamma \in \Gamma, z \in \mathbb{C}$ ) such that

(i) $\tilde{\psi}_{W}(\gamma, z)$ has the form $1+O\left(z^{-1}\right)$ for all $\gamma \in \Gamma$;

(ii) $\tilde{\psi}_{W}(\gamma, z) \in \gamma^{-1} W$ for all $\gamma \in \Gamma$.

In (i) we have introduced two abbreviations of a kind that will be used without comment from now on. By "O( $\left.z^{-1}\right)$ " we mean "some function of $z$ and possibly some other variables that vanishes as $z \rightarrow \infty$ "; and "for all $\gamma$ " means "for all $\gamma$ for which the statement makes sense" (in the present case: for all $\gamma$ such that $\gamma^{-1} W$ belongs to the big cell). The Baker function of $W$ is the function

$$
\psi_{W}(\gamma, z)=\gamma(z) \tilde{\psi}_{W}(\gamma, z)
$$

We call $\tilde{\psi}_{W}$ the reduced Baker function of $W$. The action of $\Gamma$ on $\mathrm{Gr}^{\text {ad }}$ corresponds to translation in the first variable of the reduced Baker function; more precisely, if $\eta \in \Gamma$ and $W \in \mathrm{Gr}^{\text {ad }}$, then

$$
\tilde{\psi}_{\eta W}(\gamma, z)=\tilde{\psi}_{W}\left(\eta^{-1} \gamma, z\right)
$$

(for both sides have the form $1+O\left(z^{-1}\right)$ and belong to $\eta \gamma^{-1} W$ for all $\gamma$ ).

Proposition 6.1 shows that we can restrict $\psi_{W}$ to the 1-parameter subgroup $\left\{e^{x z}\right\}$ of $\Gamma$ : the resulting function of $x$ and $z$ is called the stationary Baker function of $W$; we write it simply as $\psi_{W}(x, z)$ (rather than $\psi_{W}\left(e^{x z}, z\right)$ ). We refer to [W1] for the proof of the following.

Proposition 6.2. For any $W \in \mathrm{Gr}^{\text {ad }}, \tilde{\psi}_{W}(x, z)$ is a separable rational function of $x$ and $z$ that tends to 1 as either $x \rightarrow \infty$ or $z \rightarrow \infty$.

Here "separable" means that $\tilde{\psi}_{W}(x, z)$ is a sum of functions of the form $f(x) g(z)$. We recall at this point that the bispectral involution $b$ on $\mathrm{Gr}^{\text {ad }}$ is defined by the formula

$$
\psi_{b(W)}(x, z)=\psi_{W}(z, x) .
$$

Of course, it is not a priori clear that the right hand side of (6.2) is the Baker function of any point of $\mathrm{Gr}^{\mathrm{ad}}$; that this is the case was the main result of $\mathrm{W} 1$.

Lemma 6.3. Let $W \in \mathrm{Gr}^{\mathrm{ad}}$, and let $\Theta(x)$ be any differential operator in $x$ with coefficients that are (say) analytic on some open set in $\mathbb{C}$. Then

$$
\left(e^{-x z} \Theta(x)\right) \cdot \psi_{W}(x, z) \in e^{-x z} W \text { for all } x .
$$

\footnotetext{
${ }^{1}$ In fact it follows from Proposition 6.2 below that this set is finite
} 
After multiplication by the polynomial $m_{W}$ in (5.1), this becomes an easy exercise in the space $\mathcal{H}$ of entire functions: we leave the details to the reader.

The following consequence of Lemma 6.3 is very useful.

Corollary 6.4. Fix any $x$ such that the function $\psi_{W}(x, z)$ is regular at $x$. Then $e^{-x z} W$ is spanned by the functions

$$
e^{-x z}\left\{(\partial / \partial x)^{i} \psi_{W}(x, z)\right\}, \quad i \geq 0
$$

Proof. By Lemma 6.3, the functions (6.3) all belong to $e^{-x z} W$. They span $e^{-x z} W$ because the projection $e^{-x z} W \rightarrow \mathbb{C}[z]$ is an isomorphism, and the $i$ th function (6.3) has the form $z^{i}+$ (lower terms) for large $z$.

\section{THE ALGEBRA $\mathcal{W}$}

Proposition 6.2 shows that for large $x$ and $z$, the function $\psi_{W}$ has a series expansion of the form

$$
\psi_{W}(x, z)=e^{x z}\left\{1+\sum_{i, j=1}^{\infty} \alpha_{i j} x^{-j} z^{-i}\right\} .
$$

Formally, we can write this equation as $\psi_{W}=K_{W} \cdot e^{x z}$, where $K_{W}$ denotes the formal integral operator

$$
K_{W}=1+\sum_{i, j=1}^{\infty} \alpha_{i j} x^{-j} \partial_{x}^{-i}
$$

(from now on we write $\partial_{x} \equiv \partial / \partial x$ ). Obviously, the operator $K_{W}$ contains exactly the same information as the function $\psi_{W}(x, z)$; however, our proof of Theorem 1.4 uses these operators in an essential way, so we shall review the necessary formalism.

We denote by $\mathcal{W}$ the algebra of all formal operators of the form

$$
L(x)=\sum_{i=-\infty}^{N} \sum_{j=-\infty}^{M} \alpha_{i j} x^{j} \partial_{x}^{i}
$$

The (associative) multiplication on $\mathcal{W}$ is uniquely determined by the constraint that it should coincide with the usual one on the subalgebra $\mathbb{C}\left(\left(x^{-1}\right)\right)\left[\partial_{x}\right]$ of differential operators. The operators $K_{W}$ above are invertible elements of $\mathcal{W}$. Now let $\mathcal{F}$ be the space of formal "functions" of the form

$$
f(x, z)=e^{x z}\left(\sum_{i=-\infty}^{N} \sum_{j=-\infty}^{M} \alpha_{i j} x^{j} z^{i}\right) .
$$

The differential operators in $\mathcal{W}$ act on $\mathcal{F}$ in an obvious way, and it is easy to check that the action of $\partial_{x}$ is invertible, so that we can define the action of $\mathcal{W}$ on $\mathcal{F}$. In this way $\mathcal{F}$ becomes a free $\mathcal{W}$-module of rank 1 (as generator we can take $e^{x z}$ ).

On $\mathcal{W}$ we have the anti-automorphism $b$ defined by

$$
b(x)=\partial_{x}, \quad b\left(\partial_{x}\right)=x .
$$

On the other hand, because of the symmetry between $x$ and $z$ in the space $\mathcal{F}$, the algebra $\mathcal{W}(z)$ of formal operators in the variable $z$ also acts on $\mathcal{F}$. If $L(x)$ is an operator of the form $(7.1)$, we write $L(z)$ for the operator obtained from it by replacing $x$ by $z$ and $\partial_{x}$ by $\partial \equiv \partial / \partial z$. We then have the following simple rule of calculation. 
Lemma 7.1. For any operator $L(x) \in \mathcal{W}$, we have

$$
L(x) \cdot e^{x z}=b(L)(z) \cdot e^{x z} .
$$

As a special case of Lemma 7.1, we can reformulate the definition (6.2) of the bispectral involution in terms of the operator $K_{W}$.

Corollary 7.2. The bispectral involution on $\mathrm{Gr}^{\text {ad }}$ is characterized by the formula

$$
K_{b(W)}=b\left(K_{W}\right) .
$$

Remark. In the theory of integrable systems it is customary to work with rings of formal pseudo-differential operators in the style of Schur (see [Sch]). Readers familiar with this formalism should note that our algebra $\mathcal{W}$ is smaller than the ring $\mathbb{C}\left(\left(x^{-1}\right)\right)\left(\left(\partial_{x}^{-1}\right)\right)$ of formal pseudo-differential operators with coefficients in $\mathbb{C}\left(\left(x^{-1}\right)\right)$; for example, the element $\sum_{i=1}^{\infty} x^{i} \partial_{x}^{-i}$ of this ring does not belong to $\mathcal{W}$. As this example shows, the involution $b$ of $\mathcal{W}$ cannot be extended to the larger algebra: that is the main reason why we restrict our considerations to $\mathcal{W}$.

\section{Characterization of $\mathcal{D}(U, V)$}

In this section we work with the algebras $\mathcal{W}$ and $\mathcal{W}(z)$; we regard $\mathbb{C}(z)[\partial]$ as embedded in $\mathcal{W}(z)$ by identifying a rational function with its Laurent expansion near $z=\infty$.

Lemma 8.1. Let $U, V \in \mathrm{Gr}^{\mathrm{ad}}, D \in \mathbb{C}(z)[\partial]$. Then there is a unique differential operator $\Theta \in \mathbb{C}(x)\left[\partial_{x}\right]$ such that

$$
D(z) \cdot \psi_{U}(x, z)-\Theta(x) \cdot \psi_{V}(x, z)=e^{x z}\left\{O\left(z^{-1}\right)\right\} .
$$

Explicitly, we have $\Theta=\left[K_{U} b(D)(x) K_{V}^{-1}\right]_{+}$, where the subscript + means that we delete the terms involving negative powers of $\partial_{x}$.

Proof. Note first that

$$
D(z) \cdot \psi_{U}(x, z)=D(z) K_{U}(x) \cdot e^{x z}=K_{U}(x) D(z) \cdot e^{x z}=K_{U}(x) b(D)(x) \cdot e^{x z}
$$

(we used the rule in Lemma 7.1 at the last step). So the formula (8.1) is equivalent to

$$
\left[K_{U} b(D)(x)-\Theta K_{V}\right] \cdot e^{x z}=e^{x z}\left\{O\left(z^{-1}\right)\right\} .
$$

That is true if and only if the operator acting on $e^{x z}$ on the left has negative order; equivalently, if the operator $K_{U} b(D)(x) K_{V}^{-1}-\Theta$ has negative order. If $\Theta$ is differential, this means exactly that $\Theta=\left[K_{U} b(D)(x) K_{V}^{-1}\right]_{+}$. Since $K_{U}$ and $K_{V}$ (and hence also $K_{V}^{-1}$ ) have rational coefficients, and $b(D)$ has polynomial coefficients, it is clear that $\Theta \in \mathbb{C}(x)\left[\partial_{x}\right]$.

Proposition 8.2. Let $U, V \in \mathrm{Gr}^{\text {ad }}, D \in \mathbb{C}(z)[\partial]$. Then the following are equivalent:

(i) $D \in \mathcal{D}(U, V)$;

(ii) There is a differential operator $\Theta(x)$ such that $D(z) \cdot \psi_{U}(x, z)=\Theta(x) \cdot \psi_{V}(x, z)$;

(iii) The operator $K_{U} b(D)(x) K_{V}^{-1}$ is differential. 
Proof. The equivalence of (ii) and (iii) follows at once from Lemma 8.1. To prove the equivalence of (i) and (ii), we have only to recycle some standard arguments

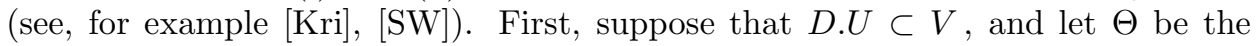
unique differential operator such that (8.1) holds, or, equivalently, such that

$$
\left(e^{-x z} D(z) e^{x z}\right) \cdot \tilde{\psi}_{U}(x, z)-\left(e^{-x z} \Theta(x)\right) \cdot \psi_{V}(x, z)=O\left(z^{-1}\right) .
$$

By Proposition 5.2, the first term here belongs to $e^{-x z} V$ for all $x$; by Lemma 6.3. so does the second term. Since generically $e^{-x z} V$ contains no function of the form $O\left(z^{-1}\right)$, it follows that the expression on the left of (3.2) is zero, that is, $D . \psi_{U}=\Theta \cdot \psi_{V}$. Conversely, suppose $D . \psi_{U}=\Theta \cdot \psi_{V}$. Differentiating with respect to $x$, we get

$$
\left\{e^{-x z} D(z) e^{x z}\right\} \cdot\left\{e^{-x z}\left(\partial_{x}^{i} \cdot \psi_{U}(x, z)\right)\right\}=e^{-x z}\left(\partial_{x}^{i} \Theta(x)\right) \cdot \psi_{V}(x, z)
$$

for all $i \geq 0$ and for all $x$. Fix any $x$ such that the functions $\psi_{U}(x, z)$ and $\psi_{V}(x, z)$ are regular at $x$. By Lemma 6.3, the right hand side of (8.3) belongs to $e^{-x z} V$. By Corollary 6.4, the functions $e^{-x z}\left(\partial_{x}^{i} \cdot \psi_{U}(x, z)\right)$ on the left hand side of (8.3) span $e^{-x z} U$; hence the operator $e^{-x z} D(z) e^{x z}$ maps $e^{-x z} U$ into $e^{-x z} V$. By Corollary 2.3 we then have $D . U \subset V$.

We shall use Proposition 8.2 in the following form.

Corollary 8.3. Let $U, V \in \mathrm{Gr}^{\text {ad }}, D \in \mathcal{W}(z)$. Then the following are equivalent: (i) $D \in \mathcal{D}(U, V)$;

(ii) the operators $D$ and $K_{U}(z) b(D) K_{V}^{-1}(z)$ are both differential.

Proof. The only thing left to see is that condition (ii) ensures that the differential operator $D \in \mathcal{W}(z)$ has rational coefficients. The argument is like that in the proof of Lemma 8.1 above: if we set $\Theta=K_{U}(z) b(D) K_{V}^{-1}(z)$ then we have

$$
D=b\left(K_{U}\right)^{-1}(z) b(\Theta) b\left(K_{V}\right)(z) .
$$

Because the Baker functions $\tilde{\psi}_{W}$ are rational in both variables $x$ and $z$, the operators $b\left(K_{W}\right)$, hence also $b\left(K_{W}\right)^{-1}$, have rational coefficients; and because $\Theta$ is differential, $b(\Theta)$ has polynomial coefficients. It follows that $D$ has rational coefficients.

\section{The action of $\widehat{G}$ on the Grassmannian}

As we observed in the Introduction, the action of the automorphisms $\Phi_{p}$ and of the anti-automorphisms $b$ and $c$ in (1.9) correspond to known symmetries of $\mathrm{Gr}^{\text {ad }}$. We have already seen this in the case of the $\Phi_{p}$ (see Proposition 5.2).

Next, we prove the crucial Theorem 1.4 from the Introduction. Using Lemma 5.3 and the definition (4.2), we see that Theorem 1.4 amounts to the following assertion.

Proposition 9.1. For any $W \in \mathrm{Gr}^{\mathrm{ad}}$, the right ideals $R_{b(W)}$ and $b\left(L_{W}\right)$ are isomorphic.

The (fractional) ideals $R_{b(W)}$ and $b\left(L_{W}\right)$ are contained (respectively) in the subalgebras $\mathbb{C}(z)[\partial]$ and $\mathbb{C}(\partial)[z]$ of the quotient field $Q$ of $A_{1}$. Since these algebras are both canonically embedded in $\mathcal{W}(z)$ (by replacing rational functions by their Laurent expansions near infinity), we can prove Proposition 9.1 by calculating in the algebra $\mathcal{W}(z)$ (rather than in $Q$ ). We shall prove the following more precise result. 
Proposition 9.2. For any $W \in \mathrm{Gr}^{\text {ad }}$, we have

$$
R_{b(W)}=K_{W}(z) b\left(L_{W}\right) .
$$

Proof. In the proof all operators are supposed to be in the variable $z$ : we omit $z$ from the notation. Applying Corollary 8.3 with $U=\mathbb{C}[z], V=b(W)$, we get that

$$
D \in R_{b(W)} \Longleftrightarrow D \text { and } b(D)\left(K_{b(W)}\right)^{-1} \text { are differential . }
$$

Also, $D \in K_{W} b\left(L_{W}\right)$ if and only if $b(D) b\left(K_{W}\right)^{-1} \in L_{W}$, so applying Corollary 8.3 again with $U=W, V=\mathbb{C}[z]$, we get

$$
D \in K_{W} b\left(L_{W}\right) \Longleftrightarrow b(D) b\left(K_{W}\right)^{-1} \text { and } K_{W}\left[\left(K_{W}\right)^{-1} D\right] \text { are differential . }
$$

But in view of Corollary 7.2, the conditions (9.1) and (9.2) coincide; hence the Proposition.

To end this section, we identify the action of the formal adjoint $c$ on $\mathrm{Gr}^{\text {ad }}$. Because the group $\widehat{G}$ is generated by the automorphisms $\Phi_{p}$ and the anti-automorphism $b$, this result is actually surplus to our needs; however, it seems of some interest in its own right.

Proposition 9.3. The action of $c$ on $\mathrm{Gr}^{\mathrm{ad}}$ is given by $c(W)=W^{*}$, where (as in W2] $W^{*}$ denotes the annihilator of $W$ with respect to the symmetric bilinear form

$$
\langle f, g\rangle=\operatorname{res}_{\infty} f(z) g(z) d z
$$

on $\mathbb{C}(z)$.

Proof. According to the definitions, we have to show that $R_{W^{*}}$ is isomorphic to $c\left(L_{W}\right)$. In fact these two right ideals coincide, for we have (denoting the formal adjoint of an operator $D$ by $D^{*}$ )

$$
\begin{aligned}
D \in R_{W^{*}} & \Longleftrightarrow D f \in W^{*} \text { for all } f \in \mathbb{C}[z] \\
& \Longleftrightarrow\langle D \cdot f, g\rangle=0 \text { for all } f \in \mathbb{C}[z], g \in W \\
& \Longleftrightarrow\left\langle f, D^{*} \cdot g\right\rangle=0 \text { for all } f \in \mathbb{C}[z], g \in W \\
& \Longleftrightarrow D^{*} g \in \mathbb{C}[z] \text { for all } g \in W \\
& \Longleftrightarrow D^{*} \in L_{W} \\
& \Longleftrightarrow D \in\left(L_{W}\right)^{*} \equiv c\left(L_{W}\right) .
\end{aligned}
$$

\section{Proof of Theorems 1.2 and 1.3}

It is now easy to see how the action of $\widehat{G}$ on $\mathrm{Gr}^{\text {ad }}$ transfers to $\mathcal{C}$. First, the formula (1.6) for the action of $\Phi_{p}$ is just the basic fact (see [W2]) that the Calogero-Moser flows and the KP flows correspond under $\beta$. Indeed, according to the definition in W2], $\beta$ maps (the conjugacy class of) a pair of matrices $(X, Y)$ to the point $W$ with reduced Baker function

$$
\tilde{\psi}_{W}(\gamma, z)=\operatorname{det}\left\{\mathrm{I}-\left(p^{\prime}(Y)-X\right)^{-1}(z \mathrm{I}-Y)^{-1}\right\},
$$

\footnotetext{
${ }^{2}$ After the change of notation $X \mapsto-X^{t}, Z \mapsto-Y^{t}$.
} 
where $\gamma(z)=e^{p(z)} \in \Gamma$. If now $q(z)$ is any polynomial with zero constant term, and we set $\eta(z)=e^{q(z)}$, then the formula (6.1) shows that $\tilde{\psi}_{\eta W}$ is obtained by replacing $X$ by $X+q^{\prime}(Y)$ in the formula (10.1); that is, we have

$$
\beta\left(X+q^{\prime}(Y), Y\right)=e^{q(z)} W,
$$

which is what the formula (1.6) states (after restoring the notation $p$ for $q$ ).

On the other hand, the bispectral involution $b$ corresponds under $\beta$ to the map $(X, Y) \mapsto\left(Y^{t}, X^{t}\right)$ on pairs of matrices. So the formula (1.7) follows from (1.6) and the fact that $\Psi_{q}=b \Phi_{-q} b$. That completes the proof of Theorem 1.2 .

For the proof of Theorem 1.3, we choose as base-point in $\mathcal{C}_{n}$ the pair $\left(X_{0}, Y_{0}\right)$ given by

$$
X_{0}=-\sum_{r=1}^{n-1} r E_{r+1, r} \quad, \quad Y_{0}=\sum_{r=1}^{n-1} E_{r, r+1}
$$

(as usual, $E_{i, j}$ denotes the matrix with $(i, j)$-entry 1 and zeros elsewhere). We shall prove something a little more precise than Theorem 1.3: namely, we shall show that any given point $(X, Y) \in \mathcal{C}_{n}$ can be moved to the base-point by applying at most three of the automorphisms $\Phi_{p}$ and $\Psi_{q}$ (in fact two are enough unless $X$ and $Y$ are both non-diagonalizable).

Lemma 10.1. Let $\boldsymbol{\lambda}=\left\{\lambda_{1}, \ldots, \lambda_{n}\right\}$ be any collection of $n$ complex numbers. Then there is a unique polynomial $p(z)$ of degree $n$ and with zero constant term such that the set of eigenvalues of the matrix $X_{0}+p^{\prime}\left(Y_{0}\right)$ is $\boldsymbol{\lambda}$.

Proof. According to Mad (Ch. 1, Sect. 2, Example 8), $\operatorname{det}\left\{X_{0}+p^{\prime}\left(Y_{0}\right)\right\}$ is equal to $n$ ! times the coefficient of $z^{n}$ in the series $\exp \{p(z)\}$. The lemma follows easily from this.

Lemma 10.2. Let $(X, Y) \in \mathcal{C}_{n}$, and suppose that $X$ is diagonalizable. Then there are unique polynomials $p$ and $q$ of degree $n$ and with zero constant term such that

$$
\Psi_{q} \Phi_{p}\left(X_{0}, Y_{0}\right)=(X, Y) .
$$

Proof. We may assume that $X=\operatorname{diag}\left(x_{1}, \ldots, x_{n}\right)$ is diagonal and that $Y$ is a Calogero-Moser matrix, that is, the off-diagonal entries of $Y$ are given by

$$
Y_{i j}=\left(x_{i}-x_{j}\right)^{-1} \text { for } i \neq j
$$

(cf. [W2], (1.14): the $x_{i}$ are necessarily distinct). By Lemma 10.1, there is a unique $p$ such that $X_{1}=X_{0}+p^{\prime}\left(Y_{0}\right)$ has the same eigenvalues as $X$; then $\left(X_{1}, Y_{0}\right)$ is conjugate to a pair $\left(X, Y_{1}\right)$ with $Y_{1}$ another Calogero-Moser matrix. Thus $Y$ and $Y_{1}$ differ only in their diagonal entries. From the non-vanishing of the Vandermonde determinant $\operatorname{det}\left(x_{i}^{j}\right)$, we find that there is a unique polynomial $q$ as in the Lemma such that $Y=Y_{1}-q^{\prime}(X)$. The Lemma follows.

Lemma 10.3. Let $(X, Y) \in \mathcal{C}_{n}$. Then there is a polynomial $r$ such that $X+r^{\prime}(Y)$ is diagonalizable.

Proof. This follows from Shiota's lemma (see W2, Lemma 5.6).

Proof of Theorem 1.9. The Theorem now follows at once from Lemmas 10.2 and 10.3 . 


\section{1. $G$ AS AN ALGEBRAIC GROUP}

Nakajima (see $\mathbb{N}$, W2 hyperkähler manifold, and that after changing the complex structure to a different one in the hyperkähler family, we obtain the space $\mathcal{C}_{n}$. It is difficult to resist the feeling that this deformation of complex structure from $\operatorname{Hilb}_{n}\left(\mathbb{A}^{2}\right)$ to $\mathcal{C}_{n}$ is related to the deformation of algebras from $A_{0}$ to $A_{1}$ (via the algebras $A_{\lambda}$ with commutation relation $[x, y]=\lambda$ ). It would be interesting to understand this analogy more precisely; here we just want to point out that considering the action of the group $G$ on $\mathcal{C}_{n}$ might lead to a new perspective on Nakajima's result.

So far we have considered $G$ simply as an abstract (discrete) group. However, the fact that it acts transitively on the affine algebraic varieties $\mathcal{C}_{n}$ suggests that we should try to view $G$ as an algebraic group in such a way that its action on $\mathcal{C}_{n}$ is algebraic. Further, from the formulae $(1.6),(1.7)$, it is clear that the action of $G$ preserves the natural holomorphic symplectic structure on the spaces $\mathcal{C}_{n}$ (see W2 , p. 9): thus $\mathcal{C}_{n}$ should be a coadjoint orbit of $G$ (or possibly of some central extension of $G$ ). The fact that these orbits are hyperkähler could then perhaps be compared to Kronheimer's result that the coadjoint orbits of finite dimensional complex semisimple groups have hyperkähler structures (see [Kr], [Ko], [B]). As N. Hitchin has pointed out to us, the phemonenon that the transitive action of $G$ on $\mathcal{C}_{n}$ becomes intransitive on $\operatorname{Hilb}_{n}\left(\mathbb{A}^{2}\right)$ then falls into perspective, since it is just what happens in Kronheimer's case.

Let us recall first that $G$ can be identified with the group $G_{0}$ of unimodularf automorphisms of the commutative algebra $A_{0} \equiv \mathbb{C}[x, y]$. Indeed, it is well known (see J]) that $G_{0}$ is generated by the automorphisms $\Phi_{p}$ and $\Psi_{q}$ defined by the formulae (1.4) and (1.5); furthermore (see [M2]) the relations between these are the same in $G_{0}$ as in $G$, so that as abstract groups we may identify $G_{0}$ and $G$. The group $G_{0}$ is a prototype example in Shafarevich's theory of infinite dimensional algebraic groups (see $\left[\mathrm{Sh}\right.$ ). The key idea is that we give $G_{0}$ a structure of infinite dimensional algebraic variety by regarding it as the union of the finite dimensional subvarieties $G^{(d)}$ of automorphisms of degree at most $d$ : here the "degree" of an automorphism $\sigma$ is defined to be the largest of the degrees of the polynomials

$$
\sigma(x), \sigma(y) ; \sigma^{-1}(x), \sigma^{-1}(y) .
$$

This idea works equally well for the group $G$ of automorphisms of $A_{1}$ : let us denote the resulting algebraic group by $G_{1}$. We claim that the algebraic groups $G_{1}$ and $G_{0}$ are not isomorphic. Indeed, Shafarevich shows in Sh] that the Lie algebra of $G_{0}$ (considered as an algebra of derivations of $A_{0}$ ) is the algebra of all (polynomial) vector fields with zero divergence on $\mathbb{A}^{2}$; that is, it is isomorphic to $A_{0} / \mathbb{C}$, where $A_{0}$ is made into a Lie algebra via the canonical Poisson bracket. A similar calculation for $G_{1}$ shows that its Lie algebra is the full algebra of derivations of $A_{1}$, so it is isomorphic to $A_{1} / \mathbb{C}$. Since their Lie algebras are not isomorphic, the algebraic groups $G_{0}$ and $G_{1}$ are not isomorphic. Thus as we deform $A_{0}$ to $A_{1}$, the complex structure on the space of ideals deforms from $\operatorname{Hilb}_{n}\left(\mathbb{A}^{2}\right)$ to $\mathcal{C}_{n}$, and the automorphism group deforms from $G_{0}$ to $G_{1}$. Certainly, $G_{0}$ acts algebraically on $\operatorname{Hilb}_{n}\left(\mathbb{A}^{2}\right)$, so it is very natural to expect at this point that $G_{1}$ should act algebraically on $\mathcal{C}_{n}$; however, that is not the case. In fact the group $G_{1}$ has no non-trivial finite dimensional homogeneous spaces; for if it did, we should obtain a

\footnotetext{
${ }^{3}$ that is, with Jacobian determinant 1
} 
non-trivial homomorphism from $A_{1} / \mathbb{C}$ to the Lie algebra of vector fields on a finite dimensional variety, and it is known that this cannot happen (see [HM], [St1]). In a similar way, we have convinced ourselves that for $n>1$ the action of $G_{0}$ on $\mathcal{C}_{n}$ is not algebraic (despite an implied belief to the contrary in [A] ).

These are discouraging facts; however, there is yet a third structure of algebraic group on $G$ that does seem to answer our purpose: namely, we can identify $G$ with a group of automorphisms of the free associative algebra $A=\mathbb{C}\langle x, y\rangle$. The abelianization map $A \rightarrow A_{0}$ induces a natural homomorphism from $\operatorname{Aut}(A)$ to $\operatorname{Aut}\left(A_{0}\right)$, and it is known that this homomorphism is bijective (see [Cz], M1]). Denoting now by $\mathcal{G}$ the subgroup of "unimodular" (that is, preserving the commutator $x y-y x$ ) automorphisms of $A$, we obtain bijective maps of algebraic groups $\mathcal{G} \rightarrow G_{0}$ and $\mathcal{G} \rightarrow G_{1}$. We claim that neither of these maps is an isomorphism; in fact the induced maps of Lie algebras are not injective. Thus $G_{0}$ and $G_{1}$ should be thought of as distinct quotient groups of $\mathcal{G}$.

It seems to us that $\mathcal{G}$ does indeed act algebraically on the varieties $\mathcal{C}_{n}$. We think that these varieties deserve further study from this point of view $\mathrm{A}$.

\section{REFERENCES}

[A] I.V. Artamkin, Action of biregular automorphisms of the affine plane on pairs of matrices, Math. USSR Izvestiya 33 (1989), 433-439.

[ACKP] E. Arbarello, C. De Concini, V. G. Kac and C. Procesi, Moduli space of curves and representation theory, Comm. Math. Phys. 117 (1988), 1-36.

[BW] Yu. Berest and G. Wilson, Classification of rings of differential operators on affine curves, IMRN 2 (1999), 105-109.

[BW1] Yu. Berest and G. Wilson, Ideals in the Weyl algebra and noncommutative projective geometry, in preparation.

[B] O. Biquard, Sur les équations de Nahm et la structure de Poisson des algèbres de Lie semisimples complexes, Math. Ann. 304 (1996), 253-276.

$[\mathrm{CH}]$ R. C. Cannings and M. P. Holland, Right ideals in rings of differential operators, J. Algebra 167 (1994), 116-141.

[Cz] A. J. Czerniakiewicz, Automorphisms of a free associative algebra of rank 2. II, Trans. Amer. Math. Soc. 160 (1972), 309-315.

[D] J. Dixmier, Sur les algèbres de Weyl, Bull. Soc. Math. France 96 (1968), 209-242.

[G] V. Ginzburg, Noncommutative symplectic geonetry and Calogero-Moser phase space, preprint (1999).

[HM] M. Hazewinkel and S. I. Marcus, On Lie algebras and finite dimensional filtering, Stochastics 7 (1982), 29-62.

[J] H. W. E. Jung, Über ganze birationale Transformationen der Ebene, J. Reine Angew. Math. 184 (1942), 161-174.

[K] K. M. Kouakou, Isomorphismes entre algèbres d'opérateurs différentiels sur les courbes algébriques affines, Thèse, Université Claude Bernard-Lyon 1 (1994).

[Ko] A. G. Kovalev, Nahm's equation and complex adjoint orbits, Quart. J. Math. Oxford Ser. (2) 47 (1996), 41-58.

[Kri] I. M. Krichever, Integration of nonlinear equations by methods of algebraic geometry, Funct. Anal. Appl. 11(1) (1977), 12-26.

[Kr] P. B. Kronheimer, A hyper-Kählerian structure on coadjoint orbits of a semisimple complex group, J. London Math. Soc. (2) 42 (1990), 193-208.

[L] L. Le Bruyn, Moduli spaces for right ideals of the Weyl algebra, J. Algebra 172 (1995), 32-48.

[Mac] I. G. Macdonald, Symmetric functions and Hall polynomials, Oxford University Press 1979; second edition 1995.

[M1] L. Makar-Limanov, On automorphism of free algebras with two generators, Funct. Anal. Appl. 4 (1970), 262-264.

\footnotetext{
${ }^{4}$ After reading an earlier version of the present paper, V. Ginzburg (see $G$ ) has shown that $\mathcal{C}_{n}$ can indeed be identified with a coadjoint orbit of a central extension of $\mathcal{G}$
} 
[M2] L. Makar-Limanov, On automorphisms of Weyl algebra, Bull. Soc. Math. France 112 (1984), 359-363.

[MR] J. C. McConnell and J. C. Robson, Noncommutative Noetherian Rings, J.Wiley \& Sons, New York, 1987.

[N] H. Nakajima, Lectures on Hilbert schemes of points on surfaces, (to appear).

[Sch] I. Schur, Über vertauschbare lineare Differentialausdrücke, Sitzungsber. Berliner Math. Ges. 4 (1905), 2-8; Gesammelte Abhandlungen Band I, Berlin-Heidelberg-New York (1973), 170176.

[Sh] I. R. Shafarevich, On some infinite-dimensional groups. II, Math. USSR Izvestija 18 (1982), $185-194$

[St1] J. T. Stafford, The Weyl algebra and finite dimensional filtering, Stochastics 14, (1984), $29-31$.

[St2] J. T. Stafford, Endomorphisms of right ideals in the Weyl algebra, Trans. Amer. Math. Soc. 299 (1987), 624-639.

[SW] G. Segal and G. Wilson, Loop groups and equations of KdV type, Publ. Math. I.H.E.S. 61, (1985), 5-65.

[W1] G. Wilson, Bispectral commutative ordinary differential operators, J. Reine Angew. Math. 442 (1993), 177-204.

[W2] G. Wilson, Collisions of Calogero-Moser particles and an adelic Grassmannian (with an Appendix by I. G. Macdonald), Invent. Math. 133 (1998), 1-41.

[W3] G. Wilson, Bispectral symmetry, the Weyl algebra and differential operators on curves, Proceedings of the V. A. Steklov Mathematical Institute 225 (to appear).

Department of Mathematics, Cornell University, Ithaca, NY 14583-4201, USA

E-mail address: berest@math.cornell.edu

Department of Mathematics, Imperial College, London SW7 2BZ, UK

E-mail address: g.wilson@ic.ac.uk 